typeset using JPSJ.sty < ver.0.8>

\title{
Effects of Nonmagnetic Impurity Doping on Spin Ladder System
}

\author{
Youichirou IINo and Masatoshi IMADA \\ Institute for Solid State Physics, \\ University of Tokyo, Roppongi 7-22-1, \\ Minato-ku, Tokyo 106
}

(Received )

\begin{abstract}
Effects of nonmagnetic impurity doping on an antiferromagnetic spin-1/2 Heisenberg ladder system are studied by the quantum Monte Carlo method. A single nonmagnetic impurity induces a localized spin-1/2 moment accompanied by static and enhanced antiferromagnetic correlations around it. Small and finite concentration of impurities induces a remarkable change of magnetic and thermodynamic properties with gapless excitations. It also shows rather sharp but continuous crossover around the concentration of about $4 \%$. Above the crossover concentration, all the spins are strongly coupled participating in the enhanced and rather uniform power-law decay of the antiferromagnetic correlation. Below the crossover, each impurity forms an antiferromagnetic cluster only weakly coupled each other. For random distribution of impurities, large Curie-like susceptibility accompanied with small residual entropy is obtained at low temperatures in agreement with recent experimental observation in $\mathrm{Zn}$-doped $\mathrm{SrCu}_{2} \mathrm{O}_{3}$. Temperature dependence of antiferromagnetic susceptibility shows power-law-like but weaker divergence than the single chain antiferromagnet in the temperature range studied.
\end{abstract}

KEYWORDS: spin ladder system, spin gap, $\mathrm{SrCu}_{2} \mathrm{O}_{3}$, impurity effect, quantum Monte Carlo method, crossover, randomness

Spin-gapped Mott insulators have recently attracted much attention since the discovery of high-temperature superconductors with pseudo-spin-gap phase near the Mott insulator. A typical theoretical model for the spingapped Mott insulator is found in the antiferromagnetic Heisenberg (AFH) model with 2-leg ladder shape (two coupled spin chains) where a large spin gap is basically due to the dominant singlet formation on the rungsi. 2) The spin gap is numerically estimated as $\Delta \sim 0.5 \mathrm{~J}$ for the uniform exchange coupling $J$. Experimentally, the ladder model is believed to be relevant for systems such as $(\mathrm{VO})_{2} \mathrm{P}_{2} \mathrm{O}_{7}$ and $\mathrm{SrCu}_{2} \mathrm{O}_{3}$. Recently, effects of Zn-doping on $\mathrm{SrCu}_{2} \mathrm{O}_{3}\left(\mathrm{Sr}\left(\mathrm{Cu}_{1-\mathrm{x}} \mathrm{Zn}_{\mathrm{x}}\right)_{2} \mathrm{O}_{3}\right)$ have been studied where $\mathrm{Zn}^{2+}$ ion substitutes $\mathrm{Cu}^{2+}(S=1 / 2)$ and plays a role of nonmagnetic impurity (3. E) Even small concentration of nonmagnetic impurities causes a drastic change of magnetic properties with disappearance of the spin gap. At low temperatures below 10K, the Zn-doping induces anomaly of the susceptibility which is perhaps due to the antiferromagnetic (AF) transition. Above the anomaly of presumable Neel temperature, Zn-doping performed for the concentration $0.01 \lesssim x \lesssim 0.08$ shows the Curie-like susceptibility which is consistent with the formation of free localized moments proportional to the number of impurities. On the other hand, the $T$-linear dependence of the specific heat is observed upon doping above $T_{\mathrm{N}}$ with the $\gamma$ value per spin similar to that of $1 \mathrm{D}$ AFH chain $(=2 / 3 J)$. The low temperature specific heat implies that the residual entropy just above $T_{\mathrm{N}}$ is inconsistently smaller than that is expected from the free localized spins with the Curie law because $J \sim 10^{3} \mathrm{~K}$ or larger. Recently exact diagonalization of clusters and the variational Monte Carlo calculations have been performed for the ladder model with regularly distributed nonmagnetic impurities. 1 It suggests quick collapse of bulk gap structure in the excitation spectra with sensitive enhancement of AF correlations for small impurity concentration consistently with experimental indications. This model has also been studied with the help of mapping to the random bond model and the appearance of the Curie-law has been suggested in three different regions of temperatures.6

In this paper, to understand these somewhat puzzling experimental results with sensitive dependence on the impurity concentration, depleted ladder system is studied using an unbiased numerical method for large system sizes. We show results of Monte Carlo calculations for the ladder model with nonmagnetic impurities in both cases of regular and random distributions of impurities. In our results, large Curie-like susceptibility and small residual entropy are consistently understood within this model, where the overlap of AF clusters formed around impurities is substantial. We also show that rather sharp crossover exists around the impurity concentration $x_{\mathrm{c}} \sim 0.04$. This is due to the fact that the overlap of AF clusters formed around the impurities becomes exponentially weak below this impurity concentration. To understand the low temperature properties for $x<x_{\mathrm{c}}$, we point out the necessity to take account of three dimensional inter-ladder coupling to explain the experimental indications even above $T_{\mathrm{N}}$.

Our model is 2-leg ladder AFH model with periodic boundary condition given by the Hamiltonian, $H=$ $J \sum_{\langle i, j\rangle} S_{i} \cdot S_{j}$, where the summation is taken for all pairs of nearest neighbor sites. To take into account the nonmagnetic impurity effect, we deplete the spins in the im- 
purity sites. For the convenience of notation, we define an index of site as $(x, y)$, where $x(y)$ is the coordinate in the leg(rung)-direction of the ladder. For instance, for $64 \times 2$-site system, $x$ is from 1 to 64 and $y$ is from 1 to 2 . The site $(x, y)$ belongs to $\mathrm{A}(\mathrm{B})$-sublattice when $x+y$ is even(odd) number.

We adopt the quantum Monte Carlo (RMC) method with an efficient loop cluster algorithm.8, 9) This algorithm together with the improved estimators reduces the statistical errors of the observables and the simulation time. We treat $64 \times 2$-site system with $0,2,4,8,10$ and 16 impurities (namely, impurity concentration $x=0$, $0.0156,0.0313,0.0625,0.0938,0.125)$, and $60 \times 2$-site system with 10 impurities $(x=0.0833)$. The lowest temperature in our simulation is $1 / 120$ in the scale of $J$. In terms of experiments in the $\mathrm{Cu}$ oxide ladders, this lowest temperature corresponds to the order of $10 \mathrm{~K}$ which is indeed relevant when one wishes to compare with experimental results. Simulations are performed with $10^{3}$-time sweeps for equilibration and $5 \times 10^{4}$-time sweeps for measurements. The autocorrelation time of the loop cluster algorithm is typically a few sweeps. In case of the random distribution of impurities, we take typically average over 72 configurations of samples.

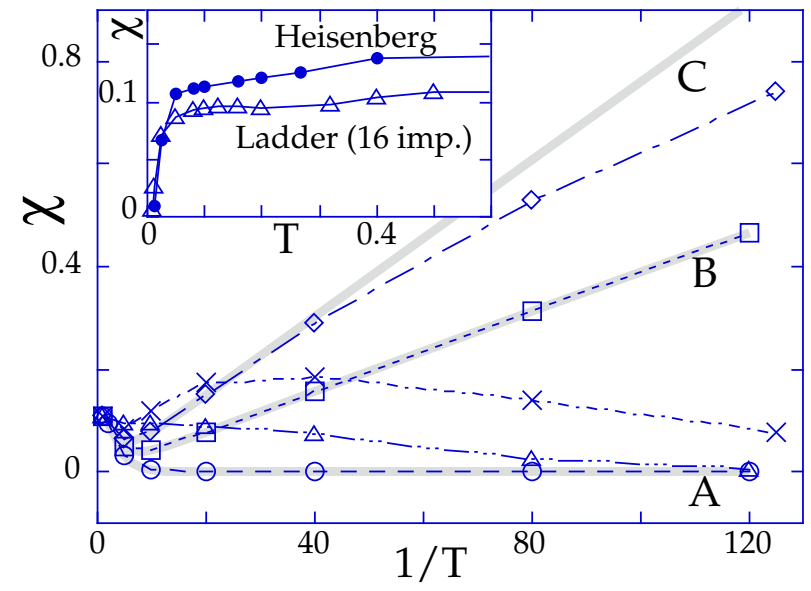

Fig. 1. 1/T-dependence of the uniform susceptibility for $0(0)$, $2(\square), 4(\diamond), 8(\times)$, and $16(\triangle)$ impurities on $64 \times 2$-site system. Lines A, B and C represent the fitting by the theoretical form. Other dashed lines are drawn as a guide to eye. The inset shows $T$-dependence of the uniform susceptibility for 64 site AFH $\operatorname{chain}(\bullet)$ and ladder system with $16 \operatorname{impurities}(\triangle)$.

First we investigate effects of nonmagnetic impurities distributed regularly, where one impurity is located at site $(1,1)$ and other impurities are put on two legs alternatingly with the same interval. In Fig.1, we show the temperature dependence of susceptibility $\chi=T^{-1} N_{s}^{-1} \sum_{i, j}\left\langle S_{i}^{z} S_{j}^{z}\right\rangle$, where $N_{s}$ is the number of sites. This figure shows the qualitative difference between 4- and 8-impurity. Without an impurity, because of the spin gap $\Delta$, susceptibility becomes zero at low temperatures in accordance with the theoretical form $\$$ $\chi(T) \sim C_{0} T^{-1 / 2} \exp (-\Delta / T)$ (Line A), where $C_{0}$ is a constant. With 2 and 4 impurities, within the temperature range we studied, the susceptibility is well described by $\chi(T) \sim C_{0} T^{-1 / 2} \exp (-\Delta / T)+C_{1}(n) / T$ (Lines B and C), where $C_{1}(n)=n / 4 N_{s}$ is the Curie constant which is proportional to the number of impurities $n$. The spin gap is estimated as $\Delta=0.497 \pm 0.001$ in the pure system while the parameter $\Delta$ is estimated as $\Delta=0.526 \pm 0.001$ and $\Delta=0.553 \pm 0.001$ for 2 - and 4-impurity cases, respectively. At $x<x_{\mathrm{c}}(\sim 0.04)$, each impurity generates one spin-1/2 moment in the sea of spin-gapped background. Because the coupling between these spin moments is weak, they behave as nearly free in this temperature range. For example, for 2 -impurity case at $1 / T=40$, the two impurities are sufficiently well separated so that we can see effects of single-impurity doping around one of it. Each isolated impurity induces $S=1 / 2$ spin. In addition it generates static correlation whose saturated value at large $\tau$ for sites $r_{0}=(1,2)$ and $r_{j}=(j, 2)$ around the impurity $(1,1)$ shows an exponential dependence on $\left|r_{0}-r_{j}\right|$ as $\left\langle S_{0}^{z}(\tau=20) S_{j}^{z}(0)\right\rangle \propto(-1)^{\left|r_{0}-r_{j}\right|} \cdot \exp \left(-\left|r_{0}-r_{j}\right| / \xi\right)$ with $\xi=2.91 \pm 0.15$, which shows good agreement with the correlation length of pure ladder system, $\xi \simeq 3.19$. Therefore it is concluded that an impurity induces $S=1 / 2$ spin as well as static and enhanced AF correlation within the range of original correlation length of the gapped system. Similar conchusions have been reached by other theoretical studies 10, 11

In contrast to this low impurity concentration, at $x>x_{\mathrm{c}}$, the susceptibility does not show the Curielaw behavior in this regular distribution of impurities. As is shown in the inset of Fig.1, it is rather similar to that in the $1 \mathrm{D}$ pure $\mathrm{AFH}$ with gapless spin excitation. (Note that the actual data suffer from finite-size effect at very low temperatures with vanishing susceptibility as $T \rightarrow 0$ ). Spins localized around impurities become strongly coupled above the crossover concentration $x_{\mathrm{c}} \sim 0.04$ in the temperature range studied.

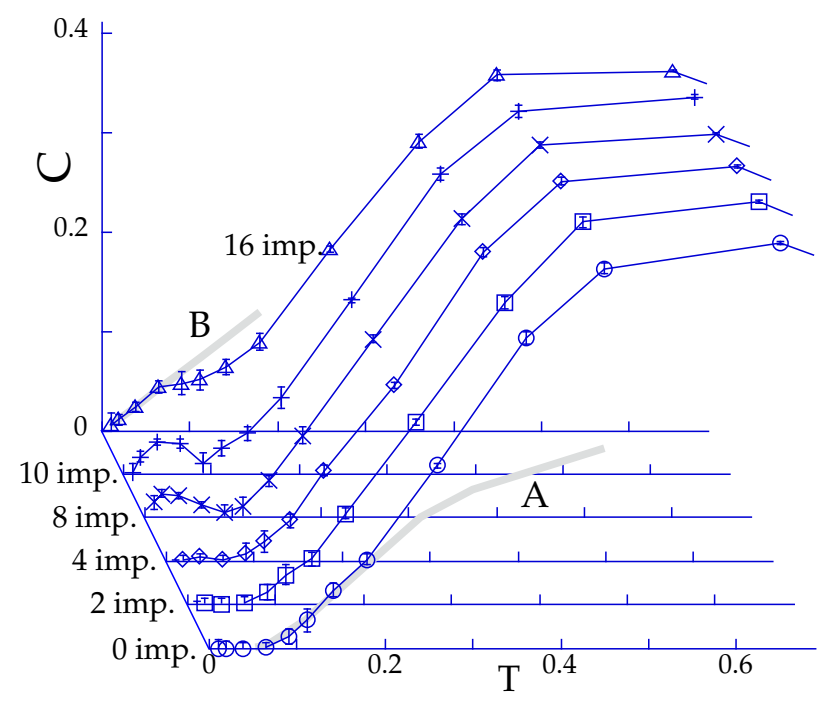

Fig. 2. T-dependence of specific heat per spin for $0,2,4,8,10$ and 16 impurities. Line A shows the theoretical form for the pure ladder at low $T$. Line B represents the specific heat per site for $1 \mathrm{D}$ AFH chain. Other lines are drawn as a guide to eye.

In Fig.2, temperature dependence of the specific 
heat per spin is presented. For the pure ladder system, if we assume the momentum dependence of the low energy triplet dispersion as $\epsilon(k)=\Delta+a \mid k-$ $\left.\pi\right|^{2}$, with $a$ being a constant, low temperature $(T \ll$ $\Delta)$ form of the specific heat $\$$ is given by $C(T)=$ $\frac{3}{4}\left(\frac{\Delta}{\pi a}\right)^{1 / 2}\left[\left(\frac{T}{\Delta}\right)^{-3 / 2}+\left(\frac{T}{\Delta}\right)^{-1 / 2}+\left(\frac{T}{\Delta}\right)^{1 / 2}\right] e^{-\Delta / T}$. This form fits well to our QMC data for $T \lesssim 0.2$, with the spin gap $\Delta$ estimated as $0.49 \pm 0.04$. At $x<x_{\mathrm{c}}(\sim 0.04)$, temperature dependence of the specific heat looks similar to the pure ladder case. For these dopings, as is seen from the data of the spin susceptibility, the systems are described by the free spins induced around the impurities in our temperature range. Similarity of $C$ to the undoped system implies that the bulk gap structure in the excitation spectrum remains unchanged. At low enough temperatures, small interaction between these localized spins is expected to yield small peak in the specific heat to release the entropy, which is beyond the temperature range of our QMC simulations.

As is seen in Fig.2, at $x>x_{\mathrm{c}}$, the specific heat at low temperatures $(T \lesssim 0.1)$ is enhanced and looks qualitatively different from the case of low doping. The low temperature tails of the specific heat appears to follow $T$-linear dependence, as is pronouncedly visible for 16impurity doping. The $T$-linear dependence is characteristic to the 1D system with gapless excitations. In fact, for $1 \mathrm{D} \mathrm{AFH}$, from the Bethe Ansatz calculation, low temperature specific heat is given as $C=(2 / 3 J) T$ t2 $)$ per site. This value fits well for the $T$-linear part observed at $x>x_{\mathrm{c}}$. It implies that below a crossover temperature $T_{\mathrm{cr}}$, all the spins become strongly coupled similarly to the $1 \mathrm{D}$ AFH model. At $x>x_{\mathrm{c}}, T_{\mathrm{cr}}$ increases more or less linearly with the doping concentration, namely, $T_{\text {cr }} \sim 0.04,0.06$ and 0.09 for $n=8,10$ and 16 , respectively. In fact, when the entropy of $n$ localized spins starts released below $T_{\text {cr }}$ with $T$-linear specific heat $C \sim(2 / 3 J) T, T_{\mathrm{cr}}$ is estimated to be $T_{\mathrm{cr}} \sim(3 J x / 2) \ln 2$. This is consistent with the numerical data for $x>x_{\mathrm{c}}$. Although the exponent of the specific heat $\alpha$ defined from $C \propto T^{\alpha}$ at asymptotically low temperatures may be different from 1 for the randomly distributed impurities, the residual entropy in our temperature range may be similar between regular and random distribution of impurities. Therefore, the specific heat at $x>x_{c}$ is consistent with the experimental observation in $\mathrm{Zn}$-doped $\mathrm{SrCu}_{2} \mathrm{O}_{3}$. The peak in the specific heat observed below $T_{\text {cr }}$ is consistent with temperature dependence of $\chi$, where localized spins become strongly coupled below $T_{\mathrm{cr}}$. From the calculated specific heat, for $\mathrm{Zn}$ doped ladder system, we predict that the specific heat linear in $T$ should decrease at $T_{\mathrm{cr}}$ when we increase temperature further before the exponential growth due to the bulk spin gap appears. In the experiments, the linear specific heat is observed even at the concentration as low as $x \sim 0.01$ around $10 \mathrm{~K}$. At least in the temperature of the order of $10 \mathrm{~K}$, we could not identify the linear specific heat for $x<x_{\mathrm{c}}$. This discrepancy may be due to the three dimensional coupling. In fact the $\mathrm{AF}$ order appears to be set in around the similar temperature.

In Fig.3, we plot $T$-dependence of equal time AF spin correlation $S(Q=(\pi, \pi), \tau=0)=N_{s}^{-1} \sum_{i, j} e^{-i Q \cdot\left(r_{i}-r_{j}\right)}\left\langle S_{i}^{z} S_{j}^{z}\right\rangle$ and AF spin susceptibility $\chi(Q=(\pi, \pi), \omega=0)=N_{s}^{-1} \int_{0}^{1 / T} d \tau$ $\sum_{i, j} e^{-i Q \cdot\left(r_{i}-r_{j}\right)}\left\langle S_{i}^{z}(\tau) S_{j}^{z}(0)\right\rangle$ for both regular and random impurity distributions.

For the regularly distributed case, in contrast to the 4-impurity case, 8-impurity case shows good coincidence with $1 \mathrm{D}$ AFH for $T \lesssim 0.1$, while $S(Q=(\pi, \pi), \tau=0)$ shows similar $T$-dependence as 0 -impurity case for 4 -impurity case. It turns out that at $x<x_{\mathrm{c}}$, spin gap nature of the ladder system is not changed, and the correlation decays exponentially in the temperature range studied. The impurity spins become coupled with presumable power-law decay of correlations only below $T_{\mathrm{cr}}$. At $x<x_{\mathrm{c}}, T_{\mathrm{cr}}$ decreases exponentially fast with decreasing $x$ and it disappears from experimentally accessible temperature range quickly because the effective exchange coupling between neighboring localized spins confined around the impurities should decrease exponentially with the decrease in the doping concentration $x$. However at $x>x_{\mathrm{c}}$, spin correlations follow the power law decay, which again indicates that all the spins become strongly coupled as in the 1D AFH chain. In fact in this regular distribution of impurities, spin correlations in real space have quantitatively similar values to the case of $1 \mathrm{D} \mathrm{AFH}$ chain with the same power $\propto 1 / r$. An important point is that in this high-doping region, not only the localized spins confined around impurities but all the spins in the system take part in this enhancement of spin correlation. This is consistent with the results of the magnetic susceptibility and the specific heat.

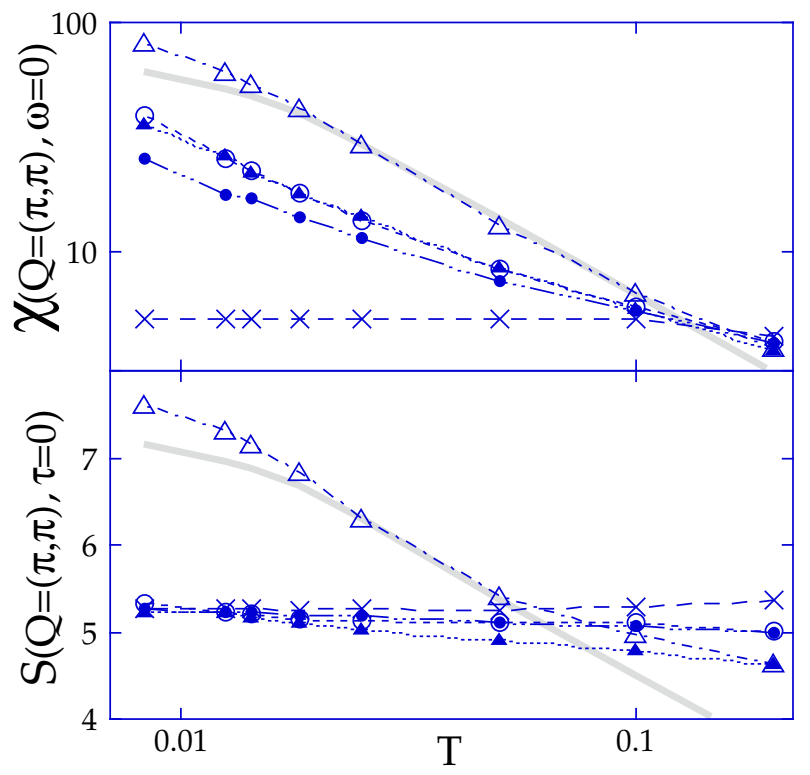

Fig. 3. Equal time spin correlation $S(Q=(\pi, \pi), \tau=0)$ and antiferromagnetic suscpetibility $\chi(Q=(\pi, \pi), \omega=0)$ are plotted for $0(\times), 4(\circ), 8(\triangle)$ impurities with regular distributions while bold curves show the case of 128 -site pure AFH chain. Filled sysmbols show the case of 4 (circle) and 8(triangle) impurities with random distributions.

Next we discuss the case of random impurity distribu- 


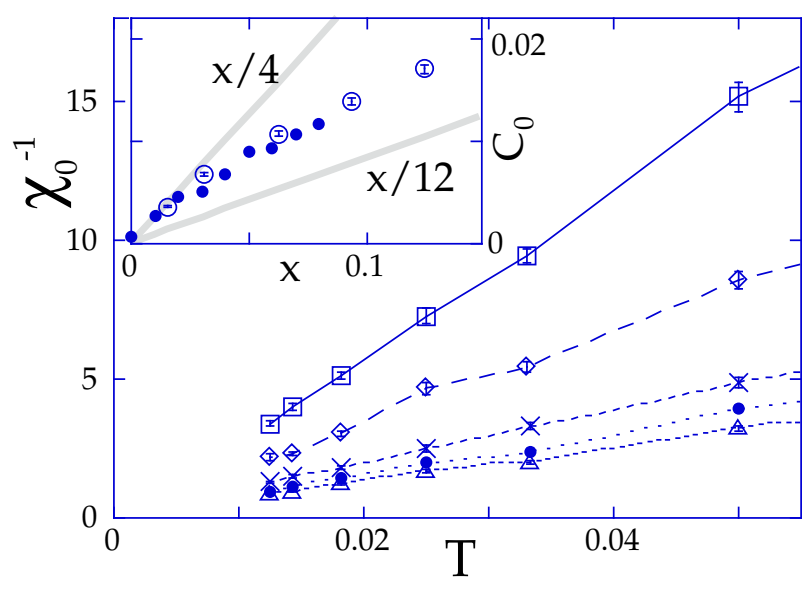

Fig. 4. T-dependence of inverse uniform susceptibility for 2( $\square)$, $4(\diamond), 8(\times), 12(\bullet)$ and $16(\triangle)$ impurities with random distribution on $64 \times 2$-site system. The inset shows the Curie constants obtained from our calculation(o) and the experiment $(\bullet)$.

tion. Residual entropy is expected to be similar to the case of regular impurity distribution in our temperature range. However, as we see in Fig.4, the uniform susceptibility has qualitatively different behavior. For all impurity concentrations, even at $x>x_{c}$, the Curie-like susceptibility is observed In fact, from the arguments by Sigrist and Furusaki $\$$ ) at $T \lesssim \Delta$ (spin gap of pure ladder system), Curie behavior of uniform susceptibility is expected with two different Curie constants $x / 4$ and $x / 12$ with a crossover between these two Curie behaviors around $T=T^{*}$. Here $T^{*}$ is the order of average coupling between impurity spins. Below $T^{*}$ all impurity spins couple with each other and the system shows the spin moment due to the fluctuating imbalance of the number of impurities on A and B sublattices. In the relevant temperature range in the experiment $\sim 0.01 J(<\Delta)$, our calculated Curie constants seem to lie between $x / 4$ and $r / 12$ and is consistent with the experimental observation.4 In terms of the argument by Sigrist and Furusaki (6) it implies that there is a wide intermediate temperature region between these two Curie behaviors.

For the case of randomly distributed impurities in Fig.3, the growths of $S(Q=(\pi, \pi), \tau=0)$ and $\chi(Q=(\pi, \pi), \omega=0)$ at low temperatures are suppressed both from the cases of regularly distributed case and the $1 \mathrm{D}$ AFH case. $S(Q=(\pi, \pi), \tau=0)$ shows very weak temperature dependence in the temperature range studied. $\chi(Q=(\pi, \pi), \omega=0)$ appears to follow approximately power-like divergence $\propto T^{-a}(a \sim 0.7)$ with weaker divergence than the case of $1 \mathrm{D}$ AFH $\left(\propto T^{-1}\right)$. Although the AF correlation is clearly enhanced from the pure ladder, its enhancement is not as large as the 1D AFH case. This numerical result does not exclude stronger enhancement of AF correlation at lower temperatures. However in the experimentally relevant temperature range $T \sim 0.01 \mathrm{~J}$, this result has shown that the argument of Nagaosa et al.13) is not applicable because they predict a more singular divergence of $\chi(Q=(\pi, \pi), \omega=0)$ than $1 \mathrm{D} \mathrm{AFH}$.

In summary, effects of nonmagnetic impurities on a spin ladder are investigated by the quantum Monte Carlo method with loop cluster algorithm. Single impurity causes the formation of a spin- $1 / 2$ localized spin confined around the impurity site. This impurity spin generates a cluster of the size $\xi \sim 3$ around it with static $\mathrm{AF}$ correlation, which is the origin of the enhancement of the AF correlation. From the results from regularly and randomly distributed impurities, a rather sharp but continuous crossover concentration $\left(x_{\mathrm{c}} \sim 0.04\right)$ is identified. Above $x_{\mathrm{c}}$, all the spins are strongly coupled with rather uniform and power-law decay of the AF correlation. Below $x_{\mathrm{c}}$, the impurity spins are only weakly coupled and is expected to give rise to power-law decay of the correlations between impurity spins only at sufficiently low temperatures. Above $x_{\mathrm{c}}$ our results are consistent with experimental results in terms of two aspects. One is a large Curie-like susceptibility with the Curie constant $\sim x$. The other is a small residual entropy and $T$-linear specific heat with $\gamma$ similar to the per spin value of $1 \mathrm{D}$ AFH chain. However, it seems to be hard to keep the same behavior in the temperature range of $0.01 J$ at $x=0.01$. We speculate that the three dimensional coupling plays an important role in keeping the similar behavior even at $x=0.01$. The AF correlation is strongly enhanced as compared to the pure ladder. However, its enhancement is not as large as the 1D AFH chain.

The authors would like to thank H. Takagi, M. Sigrist, N. Furukawa, N. Katoh and Y. Motome for enlightening discussions. This work is financially supported by a Grant-in-Aid for Scientific Research on Priority Area, "Anomalous Metallic State near the Mott Transition" from the Ministry of Education, Science and Culture. The numerical calculations were carried out using facilities of Supercomputer Center of the Institute for Solid State Physics, Univ. of Tokyo.

[1] E. Dagotto and T. M. Rice Science 271 (1996) 618, and references therein.

[2] S. R. White, R.M.Noack and D.J.Scalapino: Phys. Rev. Lett. 73 (1994) 886.

[3] M. Nohara, H. Takagi, M. Azuma, Y. Fujishiro, and M. Takano: preprint

[4] M. Azuma, Y. Fujishiro, M. Takano, T. Ishida, K. Okuda, M. Nohara and H. Takagi: preprint

[5] Y. Motome, N. Katoh, N. Furukawa and M. Imada: J. Phys. Soc. Jpn. 65, (1996) 1949.

[6] M. Sigrist and A. Furusaki: cond-mat/9606170.

[7] M. Troyer, H. Tsunetsugu and D. Würtz: Phys. Rev. B 50, (1994) 13515.

[8] H. G. Evertz, G. Lana and M. Marcu: Phys. Rev. Lett. 70, (1993) 875.

[9] U. J. Wiese and H. -P. Ying: Z. Rev. B 93, (1994) 147.

[10] G. B. Martins, E. Dagotto and J. A. Riera: cond-mat/9605069

[11] H. Fukuyama, N. Nagaosa, M. Saito and T. Tanimoto: preprint.

[12] M. Takahashi: Prog. Theor. Phys. 50, (1973) 1519.

13] N. Nagaosa, A. Furusaki, M. Sigrist and H. Fukuyama: condmat/9609016 